\title{
Influencing food preferences
}

Rats that were exposed to a 'junk food' diet in utero and as neonates before being weaned seem to prefer diets with higher levels of fat than do rats whose mothers ate standard rat chow, researchers recently reported. Further analysis suggests that exposure to this junk food diet is associated with altered development of the pathway regulating motivation and reward.

Clinical and experimental studies have shown that individuals exposed to high-fat, high-sugar diets before birth have increased risk of obesity later in life. Some research suggests that such 'maternal overnutrition' can predispose offspring to prefer highfat, high-sugar foods. The mechanisms underlying food preference, however, remain unclear.

In this study, Beverly Muhlhauser and Z.Y. Ong of the University of Adelaide in Australia assigned female rats to one of two groups, each group consisting of six rats. The first group had free access to standard rat chow (FASEB J. doi:10.1096/fj.10178392; published online 22 March 2011). The other rats had unlimited access to a junk food diet consisting of peanut butter, hazelnut spread, chocolate-flavored biscuits, extruded savory snacks, sweetened multi-grain cereal, ham- and chickenflavored processed meat and a mixture of lard and standard rat chow.

The female rats ate their respective diets for four weeks before being mated up until they weaned their offspring. After weaning, the dam and one male and one female pup from each litter were killed. The remaining pups were given free access to standard chow and to a junk-food diet. A male pup and a female pup from each litter were killed at 6 weeks of age and another male pup and female pup from each litter were killed at 3 months. The research team carried out post-mortem analyses on rats.

The rats born to dams eating junk food were significantly lighter than control offspring; this difference persisted until weaning. Additionally, rats born to mothers exposed to the junk food diets had an increased preference for fat through at least 3 months of age. The altered expression of some genes in the rats with early exposure

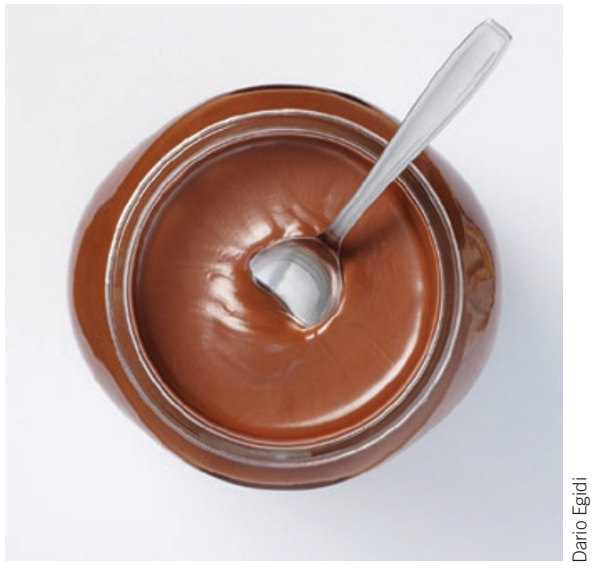

to a junk food diet was consistent with heightened activation of opioid and dopaminergic signaling within the central reward pathway, though this altered genetic expression was reversed by the time these mice reached 3 months of age. These results suggest a possible mechanism for how early life exposure to high-fat, high-sugar diets may lead to the development of childhood and adult obesity in humans.

Kirsten Dorans

\section{IDENTIFYING THE SWEET SMELL OF SUCCESS}

Olfaction - the sense of smell-may be the oldest and least well understood of the five senses. It alerts us to danger, food and other important matters and is closely linked with brain systems involved in emotion, mood, memory, learning and behavior. Stated in simple terms, our understanding of olfaction is as follows: an odorant activates neurons in the olfactory epithelium that project axons within the olfactory nerve to the olfactory bulb, activating mitral cells that in turn send information to the olfactory cortex and other neural processing areas.

Now, new research from scientists at the University of Colorado Anschutz Medical Campus (Aurora) and the University of Utah (Salt Lake City) suggests that the process is not unidirectional but rather includes a 'back and forth' interaction between the olfactory bulb and the olfactory cortex. "It was originally thought that the olfactory bulb filtered and the olfactory cortex made decisions on whether something is, for example, edible. Our study says it's not quite like that. You process information on reward in the olfactory bulb, send it to the cortex and there is a dialogue between the two. Then the brain will act," explained Diego Restrepo (University of Colorado, Aurora), who led the research, in a press release.

The study specifically examined the synchronous firing of pairs of mitral cells in the olfactory bulbs of eight mice engaged in a behavioral task in which they were challenged to learn which of two new odors was associated with a reward. The scientists aimed to determine what type of information was being transmitted by synchronized firing: odor identity or odor value. Their results indicate that synchronized firing of mitral cells conveyed information regarding odor reward (Neuron 69, 1176-1187; 2011).

These data distinguish the olfactory system from other sensory systems in a new and important way: information on value or reward is integrated into an earlier stage of neural coding. This phenomenon emphasizes the importance of context in olfactory processing.

As stated by Donald B. Katz and Joost X. Maier (Brandeis University, Waltham, MA) in an accompanying discussion of the paper (Neuron 69, 1041-1042; 2011), the new results indicate that "the dividing line between sensation and perception may be found outside the brain...[W] hile receptor neurons may respond to purely physical aspects of sensory stimuli, even the earliest stages of neural processing intrinsically pertain to what that stimulus means to the organism under current contingencies." 